\title{
Secure communication with secret sharing for the S-Network using a large set of mistrust-parties
}

\author{
Johannes Viehmann \\ Fraunhofer Institut FOKUS (MOTION), Berlin, Germany \\ Email address: \\ Johannes.Viehmann@Fokus.Fraunhofer.de (J. Viehmann)
}

\section{To cite this article:}

Johannes Viehmann. Secure Communication with Secret Sharing for the S-Network Using a Large Set of Mistrust-Parties. Advances in Networks. Vol. 1, No. 2, 2013, pp. 17-25. doi: 10.11648/j.net.20130102.11

\begin{abstract}
This article presents a solution to ensure secure communication in computer networks by using secret sharing and multiple parties mistrusting each other instead of relying on a "trusted party" or a "web of trust". In contrast to other solutions requiring asymmetric encryption, this concept can provide security even after any possible advance in cryptanalysis and even if unlimited calculation power was available to attack it. But this solution requires the computer network to have special properties. The S-Network, a trustworthy repository, is presented as a potential application. A multi-partition-routing protocol is introduced to optimize the concept for secure communication with secret sharing in the context of the S-Network.
\end{abstract}

Keywords: Secret Sharing; Trust; Mistrust; Secure Communication; PSMT; S-Network

\section{Introduction}

Secure communication is requiredfor network applications in various market sectors(e.g. eCommerce, eHealth).

The concept of secure communication with secret sharingin static computer networks with partition in mistrust-parties has been introduced in [15]. This article is an updated version of that publication, extended by describing in detail how the concept could be optimally applied for the S-Network[16], a large scale trustworthy repository for non-repudiation long term preservation.

\section{The Problem}

\subsection{Computer Networks with Strong Security Require- ments}

A secure channel between Alice and Bob is a communication channel which allows them to exchange messages in a finite time so that the secrecy, integrity and authenticity of the messages can be ensured and that the temporal order in which the delivered messages were sent by Alice can be reconstructed by Bob. There are provable secure solutions to keep perfect secrecy [12], but integrity, authenticity and the order can only be ensured with arbitrary high probability: Bit sequences passing tests for these could be guessed.

To enable secure communication in a computer network, any two participants should be able to establish a secure channel with each other. The needed level of security variesfrom application to application. Strong long term security means, that the cryptographic concept should be secure and practically useable for the future - independent from any possible further technical development. For the following, strong long term security requirements are assumed.

\subsection{Providing Secure Channels in Big Networks is Diffi- cult}

It is possible to build an arbitrarily secure channel between any two participants Alice and Bob having strong long term security properties, but that requires that Alice and Bob share an exclusive secret in advance. Alice and Bob have to check their identities and exchange the secret manually.In a small network with only a few participants, it is possible to do such a manual procedure for all possible pairs of participants. But the effort grows quadratically with the number of participants. With several thousand or with several million participants, this is not manageable.

\section{State of the Art}

\subsection{Secure Communication with "Trusted Party"}

"Trusted parties", sometimes called "trusted third parties", can be used to provide secure communication between any two participants in computer networks. The idea is that all participants identify themselves only to the "trusted party". 
For the further usage of the "trusted party" there are different concepts:

Inline usage of a "trusted party": Each participant shares an exclusive key with the "trusted party" so that a secure channel can be built between each participant and the "trusted party". Messages between two simple participants Alice and Bob are first send from Alice to the "trusted party" over a secure channel and then the "trusted party" forwards them to Bob over another secure channel. All messages have to pass the "trusted party", which makes it likely that the central "rusted party" becomes a bottleneck.

Online usage of a "trusted party" as key server: The "trusted party" generates a session key for Alice and Bob so that they can build a direct secure channel between them. See [9] for a solution with this approach. With keys of constant length, this approach reduces the workload of the "trusted party".

At least somehow offline usage of a "trusted party" or of a hierarchy of several "trusted parties" as certification authority (CA): See [7] for a description of public key infrastructures (PKI) and a discussion of the advantages in comparison with a key server. Most importantwith regard to strong long term security requirement is, that asymmetric encryption (e.g.[10]) is required for PKI solutions.Eventually in the future any asymmetric algorithms can be broken in a relevant short time. For algorithms whose security depends on the assumed difficulty of calculating discrete logarithms or to do prime factorization for large numbers, a theoretical solution for breaking them with quantum computers in polynomial time has already been shown in [13]. For the prime factorization of small numbers, it has been shown that the Shor algorithm really works [8].

Potentially insecure functions used for creating signatures on certificates are another potential point to attack a PKI. See [14] for an attack that takes advantage of the MD5 cryptographic hash function that has been widely used on certificates, but which is not collision resistant.Public keys are typically used to encrypt and exchange symmetric session keys so that messages can be encrypted with more efficient symmetric cyphers like AES [5](i.e. hybrid encryption). However, if the security of the symmetric cypher used for hybrid encryption might eventually be broken, thisis an additional vulnerability. Recent advances in cryptanalysis [3] show that this threat should be taken serious.

So in a typical PKI, there are at least three different potentially insecure algorithms that can be attacked independently. It is enough to break just one of these potential weaknesses to break the entire system's security.

No matter how "trusted parties" are used - the security of the communication depends on the fair and always correct behavior of the "trusted party". Why should participants trust the "trusted party"? To control institutions that have so much power is difficult and maybe it is utopian or naive to believe that universal neutrality can at all be enforced in a big network that really matters.

\subsection{Secure Communication with "Web of Trust"}

To avoid the need to trust in some single party, the "web of trust" offers a decentralized alternative concept [4]. However, with this approach, it is not possible to achieve legal validity and it requires asymmetric encryption, too. Furthermore, the demands for the users are high as they have to decide whom to trust.

In general, it has also to be questioned whether trust is transitive at all.

\subsection{Secure Communication with Secret Sharing}

Secret sharing is a technology to split a secret $x$ into a set of $n$ pieces with the property that you need at least $t$ pieces of the set to be able to reconstruct $x$ from that subset. Any subset with less than the threshold $t$ pieces does not reveal any information about $x$ at all. There are several perfectly secure secret sharing systems known, e.g.[11].

Secret sharing can be used to avoid the need to trust a single party by dividing the responsibility for trust related things between several parties. A typical application of secret sharing is to store a secret, for example a secret key.

It is also possible to use secret sharing for "perfectly secure message transmission" (PSMT) over disjoint paths as shown in [6]. These solutions require a set of separated communication channels (called "wires") between sender and addressee. But how these disjunct "wires" could be realized is not mentioned, neither how the identities could be checked nor how authentication could work.In [1] a method to find separate wires is presented, but it provides only paths with disjunct edges, not with disjoint nodes. Hence, it is not a solution for PSMT.

\section{Basic Notation and Requirements}

Let $x$ and $y$ be bit sequences. The concatenation of $x$ and $y$ prefixed with their identifiers and lengths is noted as $x \circ y$.The symmetric encryption of a bit sequence $x$ with key $K$ is notated as $\mathrm{E}_{K}(x)$. The corresponding decryption is written as $\mathrm{D}_{K}\left(\mathrm{E}_{K}(x)\right)$. Let $\mathrm{P}(x)$ be a function calculating a message authentication code (MAC) of a bit sequence $x . K, \mathrm{E}_{K}(x)$, $\mathrm{D}_{K}\left(\mathrm{E}_{K}(x)\right), \mathrm{P}(x)$ and $x \circ y$ are bit sequences. Messages are bit sequences, too.

The following notation willbe used for the set of pieces of a secret sharing split:

$$
\mathrm{Z} n_{t}(x)=\mathrm{T} n_{t, 0}(x), \ldots, \mathrm{T} n_{t, n-1}(x)
$$

The inverse operation will be noted as:

$$
x=\mathrm{Z} n_{t}^{-1}(M) \mid M \subseteq Z n_{t}(x) \wedge \# M \geq t
$$

The concept introduced in this article makes use of security technologies like secret sharing that do have a threshold. To describe a unique security level for the entire system, a constantthreshold $\Psi$ is defined. $\Psi$ is a natural number and it must be greater than two.

\subsection{S-Nodes with Partition in Mistrust-Parties}

The concept for secure communication presented in this 
paper requires an applicable legal framework and it requires the computer network in which the secure communication takes place to have the following properties:

The logical addresses of the logical systems within the network must be everlasting, absolute and unique. In the following, such a uniquely addressable logical system will be called an S-Node.S-Nodes added to the network have to be kept accessible by their logical addresses. If an $\mathrm{S}$-Node is not accessible because of some failure, it has to be repaired and restored within a finite time. Such a network may be called a static network.

For each S-Node there must be exactly one natural or juristic person responsible for it in a legal sense: the $\mathrm{S}$ Operator.Let $X$ be the set of all S-Operators in a static network. A partition of such a static network is the split of $X$ into not empty disjoint subsets so that the union of all subsets is $X$. The subsets of a partition of a static network are called parties.

The solution presented in this paper requires a special partition of the static network with at least $\Psi$ parties so that any two S-Operators belonging to two different parties mistrust each other in a way that they will not cooperate for illegal and therefore potentially dangerous manipulations. Such a partition is called a partition into mistrust-parties.

This mistrust between the parties can be established by a strict geographical, cultural and legal separation, by laws that prohibit certain forms of cooperation explicitly and by active measures to test the correct behavior of the $\mathrm{S}$ Operators in the sense of these laws. Such a test can include fake proposals for building manipulative coalitions, for example. S-Operators have the duty to report illegal offers they get in a standardized fashion. Because any illegal offer could just be a fake for testing the correct reaction, not reporting them might be very risky. Details about the concept of creating trust with a set of mistrust-parties and its application for the S-Network can be found in [16].

MP is used as abbreviation for mistrust-party in general. A certain MP is identified with an index $i$ and noted as $M P_{i}$. If an $\mathrm{S}$-Operator belongs to $M P_{i}$, all the $\mathrm{S}$-Nodes he is responsible for belong to $M P_{i}$, too. Let $\# M P_{i}$ be the total number of S-Nodes belonging to mistrust-party $M P_{i}$.

\section{Solution with Secret Sharing and MPs}

\subsection{Acquaintances, Partisan Forwarding}

Two S-Nodes are called acquaintances, if messages can be exchanged between them over an arbitrary secure channel. Therefore the $\mathrm{S}$-Operators of the acquaintances have to check the identities of each other's S-Node's owner and they have to exchange the necessary communication data (including an exclusive secret key). This security critical manual operation is a high effort.

The S-Operators do also have to make sure that data can actually be transmitted between acquaintances in a finite time. Therefore, S-Operators of two S-Nodes that are acquaintances have to negotiate manually appropriate physical channels and they have to provide them to the S-Nodes. For example, one channel could be a direct microwave transmission and the Internet could be used as another single channel between the acquaintances.

Acquaintances do have high responsibility for each other. In order to split responsibilities between $\Psi$ MPs, an arbitrary S-Node $S_{x}$ must get for each mistrust-party $M P_{i}$ at least one $\mathrm{S}$-Node belonging to $M P_{i}$ as acquaintance. This ensures that the identity of the owner of $S_{x}$ has to be verified for each $M P_{i}$ at least by one $\mathrm{S}$-Operator belonging to that $M P_{i}$ whose $\mathrm{S}$ Node becomes an acquaintance.

Because of the high manual effort, an S-Node cannot have more than just a few acquaintances to be practicable.

Only acquaintances may communicate directly with each other. If two $\mathrm{S}$-Nodes are not acquaintances, a message $m$ can be exchanged between them if there is a series of pairwise acquaintances among them and if $m$ can be forwarded from one acquaintance to another along that series. Such an indirect connection is called a forwarding-connection. The forwarding $\mathrm{S}$ - Nodes between the sender and the addressee are called forwarders.For the solution presented here, any two $\mathrm{S}$-Nodes must be acquaintances or there must be a forwarding-connection between them.

In contrast to the direct communication with an acquaintance, the forwarding communication cannot take place over a secure channel because a sender and an addressee who are not acquaintances do not have an exclusive shared secret key - they do not even know whether their pretended communication partner exists at all.

To make the communication between $\mathrm{S}$-Nodes which are not acquaintances secure and reliable, there are additional requirements. For any two S-Nodes $S_{A}$ and $S_{B}$ belonging to the same $M P_{i}$, there must be a connection without any $\mathrm{S}^{-}$ Node of all the other MPs involved. This means, that if $S_{A}$ and $S_{B}$ are not acquaintances, there must be a forwarding-connection between them so that all the forwarders belong to $M P_{i}$. Such a connection within a single MP is called partisan forwarding.

If the network structure within $M P_{i}$ is like a single ring so that each $\mathrm{S}$-Node belonging to $M P_{i}$ has exactly two acquaintances in $M P_{i}$, there is always a partisan forwarding between any two $\mathrm{S}$-Nodes belonging to $M P_{i}$.

\subsection{Partition-Routing}

The following protocol for partition-routing enables secure communication between any two S-Nodes $S_{A}$ and $S_{B}$ that are not acquaintances:

Preparation: Let $x$ be the bit sequence to be transmitted. $S_{A}$ creates a bit sequence $x_{p}$ containing a random one-time key $K_{R}$, the encryption $\mathrm{E}_{K R}(x)$ and a message authentication code $\mathrm{P}\left(K_{R}^{\circ} x\right)$.

$$
x_{p}=K_{R}^{\circ} \mathrm{E}_{K^{R}}(x) \circ \mathrm{P}\left(K_{R}^{\circ} x\right)
$$

Let $n$ be $n \in \mathbb{N} \mid n \geq \Psi$. $S_{A}$ splits $x_{p}$ with secret sharing:

$$
\mathrm{Z} n_{\Psi}\left(x_{p}\right)=\left\{\mathrm{T} n_{\Psi, 0}\left(x_{p}\right), \ldots, \mathrm{T} n_{\Psi, n-1}\left(x_{p}\right)\right\}
$$


Let $A_{B}$ be the address of the addressee $S_{B}$. Let $H$ be additional required header data (e.g. some message number and the current time). $S_{A}$ generates $n$ split messages $\tau_{i}$ :

$$
\tau_{i}=A_{B}^{\circ} H \circ \mathrm{T} n_{\Psi, i}\left(x_{p}\right)
$$

Separation: $S_{A}$ sends each $\tau_{i}$ over a secure channel to a different acquaintance of $S_{A}$ not belonging to any of the MPs $S_{A}$ or $S_{B}$ belongs to. $S_{A}$ may not send more than one piece of $\mathrm{Zn} n_{\Psi}\left(x_{p}\right)$ into any MP.

Check and forwarding: Each forwarding S-Node $S_{f}$ decrypts and checks messages $m$ arriving over secure channels from its acquaintances.

If $m$ is from an acquaintance not belonging to the same MP as $S_{f}$, this acquaintance is the sender $S_{A}$. $S_{f}$ generates an identity confirmation $I_{A i}$ containing the address of $S_{A}$, and additional identity data that was manually exchanged and verified when $S_{A}$ and $S_{f}$ became acquaintances. $S_{f}$ adds $I_{A i}$ as proof of authenticity to $m$ :

$$
m=\tau_{i} \circ I_{A i} .
$$

Else if $m$ is from an acquaintance belonging to the same MP as $S_{f}, m$ must already contain an $I_{A i}$.

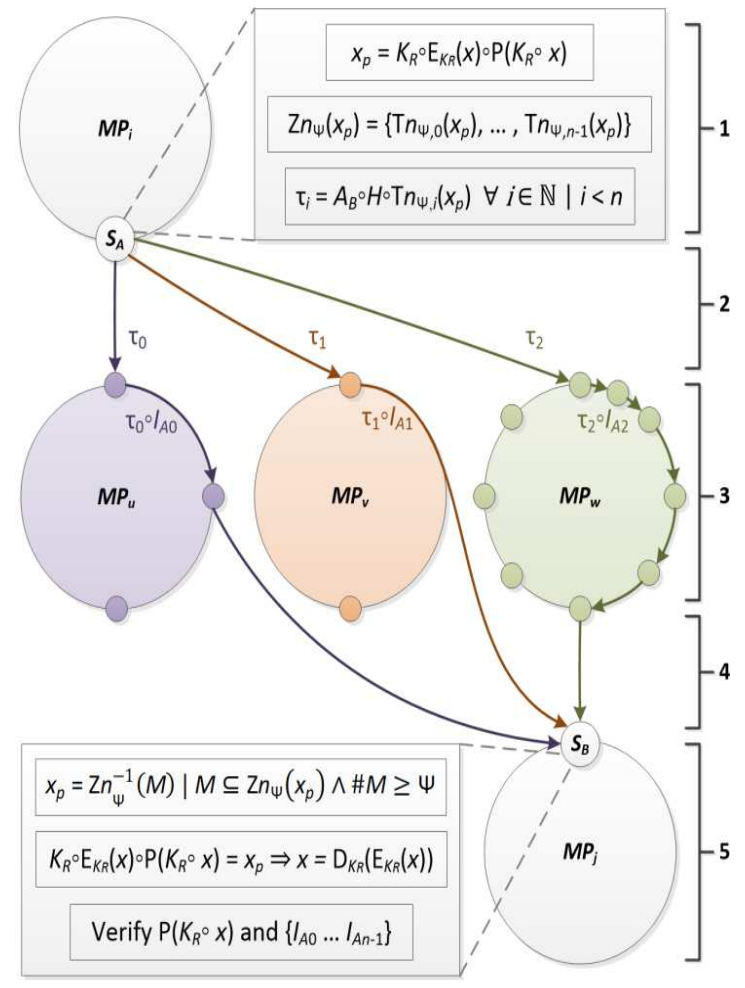

Figure 1. Partition-routing with $\Psi=n=3 . M P_{i}$ may stand for the same $M P$ as $M P_{j}$, but $M P_{w}, M P_{v}$ and $M P_{w}$ have to be distinct mistrust-parties.

$S_{f}$ must forwardcorrect messages $m$ for an addressee $S_{B}$ according to these rules:

If $S_{B}$ is not an acquaintance of $S_{f}, S_{f}$ forwards $m$ over a secure channel to the next forwarder, who must be one of the acquaintances of $S_{f}$ belonging to the same MP as $S_{f}$. The forwarder must be chosen so that $m$ gets closer to an acquaintance of $S_{B}$.

Continue with step 3 ) for the next forwarder.
Else if $S_{B}$ is an acquaintance of $S_{f}$, $S_{f}$ forwards m over a secure channel to $S_{B}$. Continue with step 4).

Check and collection: The addressee $S_{B}$ decrypts and checks messages arriving over secure channels from its acquaintances and extracts $\operatorname{T} n_{\Psi, i}\left(x_{p}\right)$ from $\tau_{i}$ if possible. Correct arriving parts $\mathrm{T} n_{\Psi, i}\left(x_{p}\right)$ and the according identity confirmations $I_{A i}$ are collected and stored together with the information from which MP they actually were forwarded.

Reconstruction and final check: As soon as at least $\Psi$ parts $\operatorname{T} n_{\Psi, i}\left(x_{p}\right)$ of the set $\mathrm{Z} n_{\Psi}\left(x_{p}\right)$ arrived correct at the addressee $S_{B}$ together with the confirming $I_{A i}$ from $\Psi$ different MPs, $S_{B}$ can try to reconstruct $x_{p}$ from that subset of $\operatorname{Zn} n_{\Psi}\left(x_{p}\right)$.

The original data $x$ can be decrypted with $K_{R}$ :

$$
x=\mathrm{D}_{K^{R}}\left(\mathrm{E}_{K^{R}}(x)\right) .
$$

The integrity can be checked with $K_{R}, x$ and $\mathrm{P}\left(K_{R}{ }^{\circ} x\right)$.

Only the sender $S_{A}$ and the addressee $S_{B}$ do get more than one piece of $Z n_{\Psi}\left(x_{p}\right)$ if this protocol is followed properly: In step 2, all the parts $\mathrm{T} n_{\Psi_{i}}\left(x_{p}\right)$ are distributed over secure channels to different MPs. The forwarding of the loop in step 3)a) between an acquaintance of $S_{A}$ and an acquaintance of $S_{B}$ is a strictly partisan forwarding over secure channels. This means that all the parts $\operatorname{T} n_{\Psi i}\left(x_{p}\right)$ stay in exactly the MP they were sent to at step 2 until they reach an acquaintance of $S_{B}$. Only then, at step 3)b), all the parts are send to the same MP, but they are directly send over secure channels to the addressee $S_{B}$.

To reconstruct $x_{p}$ from a subset of $Z n_{\Psi}\left(x_{p}\right)$, at least threshold $\Psi$ parts of $\operatorname{Zn} n_{\Psi}\left(x_{p}\right)$ are required. Any attack to get $x_{p}$ and therefore any manipulation being more sophisticated than just guessing an entire valid bit sequence must affect at least $\Psi$ forwarders in $\Psi$ different MPs.

The identity confirmation $I_{A i}$ as proof of authenticity has to be identical from at least $\Psi$ different MPs, too. To cheat requires again that at least $\Psi$ S-Nodes in $\Psi$ different MPs behave incorrect.

\subsection{Optimization}

With the simple ring like network structure and each SNode having exactly two acquaintances belonging to the same MP, this is not yet a practicable solution:

The efficiency is unusably low. Partisan forwarding would need great many S-Nodes as forwarders. In the worst case, a message has to be forwarded by $50 \%$ of the S-Nodes that belong to $M P_{i}$. With thousands or millions of S-Nodes, this would be terribly slow.Messages are not just forwarded - they have to be decrypted, checked and encrypted with another key. On average each S-Node would have to process about $25 \%$ of all the messages exchanged by forwarding through its MP.

The total system robustness would be low. If only two SNodes belonging to the same MP are temporary not reachable for their acquaintances, the entire ringlike network structure would break into two separate segments $R$ and $Q$ so that any partisan forwarding between an S-Node in $R$ and another $\mathrm{S}$-Node in $Q$ would fail.

Robustness against failures in a communication network 
can be increased by mashing up the network tighter with additional redundant connection possibilities so that alternative routes can be chosen in case of failures [2].By increasing the number of acquaintances within the same MP per S-Node, alternative routes for the partisan forwarding can be created. But that implies also a higher manual effort.

With a few more carefully chosen acquaintances for each S-Node and with a fitting routing concept, a good robustness can be achieved. By doing so, the length of the most efficient partisan forwarding between any two S-Nodes belonging to the same MP can be reduced to a practical value, too. In[15], a decentralized optimization creating well distributed acquaintances in an iterative procedure is shown in detail. Only results are summarized here.

The optimization requires the static address of an S-Node to consist of two components - one identifying the mistrust-party $M P_{i}$ the S-Node belongs to and the other identifying the $\mathrm{S}$-Node within $M P_{i}$. The last is called the Intra-MP-Address. The Intra-MP-Address must be a natural number and it must be unique within its mistrust-party.

For the optimization, for each S-Node $S_{x}$ belonging to $M P_{i}$, two acquaintances belonging to the same $M P_{i}$ are chosen according to the following rules:

The S-Node with the biggest Intra-MP-Address in $M P_{i}$ smaller than the Intra-MP-Address of $S_{x}$ becomes an acquaintance of $S_{x}$, if such an S-Node exists.

The $\mathrm{S}$-Node with the smallest Intra-MP-Address in $M P_{i}$ bigger than the Intra-MP-Address of $S_{x}$ becomes an acquaintance of $S_{x}$, if such a $\mathrm{S}$-Node exists.

Additionally, the $\mathrm{S}$-Node belonging to $M P_{i}$ with the smallest Intra-MP-Address in $M P_{i}$ and the S-Node belonging to $M P_{i}$ with the biggestIntra-MP-Address in $M P_{i}$ become acquaintances.

The result is again a ringlike network structure per MP, but the $\mathrm{S}$-Nodes on that ring are now sorted by their Intra-MP-Address. The ring-distance $\mathrm{R}\left(S_{A}, S_{B}\right)$ between two SNodes $S_{A}$ and $S_{B}$ belonging to the same $M P_{i}$ is the number of S-Nodes on the sorted ring that are between $S_{A}$ and $S_{B}$ in the shorter direction.

Let $d$ be a natural number bigger than one. Each S-Node $S_{x}$ belonging to $M P_{i}$ should additionally have those S-Nodes as acquaintances that have a ring-distance of $\left(d^{f}-\right.$ 1) with $f \in \mathbb{N} \wedge f<\left\lceil\log _{d}\left(\# M P_{i}\right)\right\rceil$ to $S_{x}$. Because the ring-distances might change whenever a new $\mathrm{S}$-Node is inserted into $M P_{i}$ and making new acquaintances has a high manual effort, for this optimization an approximation to the perfect distribution with enduring well-chosen acquaintances is the best solution.

If each S-Node has these acquaintances then in the optimized partisan forwarding process of a message between two arbitrary S-Nodes $S_{A}$ and $S_{B}$ belonging to the same $M P_{i}$, at each forwarding step from $S_{\text {old }}$ to $S_{\text {new }}$ the ring-distance to $S_{B}$ is reduced according to this formula:

$$
R\left(S_{\text {new }}, S_{B}\right) \leq R\left(S_{\text {old }}, S_{B}\right)-\frac{R\left(S_{\text {old }}, S_{B}\right)}{d}
$$

Let $F_{i}$ be the number of $\mathrm{S}$-Nodes required as forwarders between $S_{A}$ and $S_{B}$ in an optimized partisan forwarding in $M P_{i} . F_{i}$ would then be logarithmic with the number of $\mathrm{S}$ Nodes belonging to $M P_{i}$ :

$$
F_{i} \leq(d-1) *\left\lceil\log _{d}\left(\# M P_{i}\right)\right\rceil
$$

Let $A_{i}$ be the number of acquaintances each S-Node $S_{x}$ needs in his own MP to provide such an efficient partisan forwarding. The upper bound of $A_{i}$ for this optimization is:

$$
A_{i} \leq 2 *\left\lceil\log _{d}\left(\# M P_{i}\right)\right\rceil
$$

For $d=2, F_{i}$ becomes minimal, but $A_{i}$ becomes maximal, so the most acquaintances per $\mathrm{S}$-Node will be required. Because making many acquaintances means a high manual effort, it probably makes sense to choose a higher $d$ and to accept slightly longer routes in the partisan forwarding.

\subsubsection{Foresighted Partisan Forwarding}

In the process of partisan forwarding each forwarder SNode being not an acquaintance of the addressee $S_{B}$ has to identify the acquaintance that would be the next optimal forwarder. In a network constructed the way shown before, that is the acquaintance with the Intra-MP-Address having the lowest address-distance to the Intra-MP-Address of $S_{B}$.

If some S-Node $S_{F}$ would be the next optimal forwarder, but the current forwarder $S_{E}$ cannot reach $S_{F}$, alternative routes may be tried until $S_{F}$ is restored and reachable again. Alternative routes are not necessarily less efficient. To find the best alternative route is however more difficult: the address-distances have to be checked further ahead.

Let $X$ be a set of S-Nodes that belong to $M P_{i}$. Let $\mathrm{B}(X)$ be the set of those $\mathrm{S}$-Nodes belonging to the same $M P_{i}$ which have at least one acquaintance in set $X$.For optimal routing, $S_{E}$ has to choose the S-Node in $\mathrm{B}\left(\mathrm{B}\left(\left\{S_{E}\right\}\right) \backslash S_{F}\right) \backslash S_{E}$ as next forwarder that has the Intra-MP-Address with the minimal address-distance to the Intra-MP-Address of $S_{B}$.

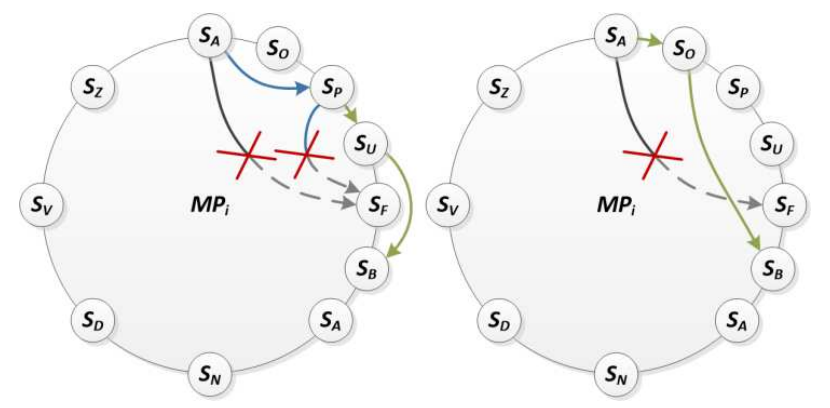

Figure 2. If some S-Node $S_{F}$ is not reachable, foresighted partisan forwarding (right side) involving $S_{O}$ is more efficient than forwarding to the acquaintance $S_{P}$ with the lowest ring-distance to the addressee $S_{B}$ (left side).

Let $S_{U}$ be an acquaintance of the addressee $S_{B}$. If the addressee $S_{B}$ is not reachable for $S_{U}$, other acquaintances of $S_{B}$ may be tried instead. Each $\mathrm{S}$-Node in $\mathrm{B}\left(\left\{S_{B}\right\}\right) \backslash S_{U}$ that has not yet been tried can be chosen as a preliminary target for the partisan forwarding route. The number of alternative acquaintances for each $\mathrm{S}$ - Node in $M P_{i}$ is between $\left\lceil\log _{d}\left(\# M P_{i}\right)\right\rceil-1$ and $\left\lceil\log _{d}\left(\# M P_{i}\right)\right\rceil * 2-1$.

To avoid endless circling messages all alternative for- 
warders have to be logged in the message's header.

\subsection{Acquaintances in Foreign MPs}

In the partition-routingprotocol between the first and the last forwarder only partisan forwarding is used to deliver each split message from the sender $S_{A}$ to the addressee $S_{B}$. For an efficient routing, it is essential to find an acquaintance of $S_{B}$ belonging to the MP in which the entire partisan forwarding takes place so that it can be used as preliminary target in the optimized partisan forwarding process.

Therefore, those S-Nodes in different MPs that have the same Intra-MP-Address should become pairwise acquaintances.If in any $M P_{i}$ there is an $\mathrm{S}$-Node $S_{i \chi}$ with the Intra-MP-Address $\chi$, but in another $M P_{j}$ there is not yet an $\mathrm{S}$ Node having the same Intra-MP-Address $\chi, S_{i \chi}$ must get some suboptimal preliminary acquaintance in $M P_{j}$ if $S_{i x}$ needs at least one foreign acquaintance in $M P_{j}$.

Let $S_{j \varphi}$ be the S-Node in $M P_{j}$ having the greatest Intra-MP-Address smaller than $\chi$. Then $S_{j \varphi}$ becomes the suboptimal preliminary acquaintance of $S_{i \chi}$ in $M P_{j}$. If later an SNode $S_{j \chi}$ is added to $M P_{j}$, then $S_{j \chi}$ becomes the optimal acquaintance of $S_{i \chi}$ in $M P_{j}$. The suboptimal preliminary acquaintance $S_{j \varphi}$ becomes superfluous for $S_{i \chi}$.

\subsubsection{Protocol for Optimized Partition-Routing}

Let $S_{i \alpha}$ be the sender belonging to $M P_{i}$. Let $S_{j \beta}$ be the addressee belonging to $M P_{j}$ having the Intra-MP-Address $\beta$.

The following protocol has to be repeated for each split message of the partition-routing protocol. It delivers such a message $m$ from $S_{i \alpha}$ to $S_{j \beta}$. All the forwarders must belong to the same MP. Let $M P_{v}$ be that MP. Let $S_{v^{*}}$ be a variable for an S-Node belonging to $M P_{v}$.

Check for common acquaintance: $S_{i \alpha}$ sends $m$ to an acquaintance $S_{v^{*}}$ belonging to $M P_{v}$. If $S_{v^{*}}$ is also an acquaintance of $S_{j \beta}$ continue with step 4).

Route to optimal acquaintance: With the foresighted partisan forwarding, the S-Nodes in $M P_{v}$ try to deliver $m$ to an $\mathrm{S}$ - Node $S_{v \beta}$ belonging to $M P_{v}$ and having the same Intra-MP-Address $\beta$ as $S_{j \beta}$. If the S-Node $S_{v \beta}$ exists and can be reached continue with step 4 ).

Go to start point for alternative search loop: $S_{v^{*}}$ is set to the $\mathrm{S}$-Node having the biggest Intra-MP-Address smaller than $\beta$ in $M P_{v}$.If the foresighted partisan forwarding did not end at $S_{v^{*}}$, but at $S_{v \chi}, m$ must be send now to $S_{v^{*}}$. This should always be possible in a single forwarding step because $S_{v^{*}}$ and $S_{v \chi}$ are at least acquaintances.

Try to reach addressee: If $S_{v^{*}}$ is an acquaintance of $S_{j \beta}, m$ is forwarded to $S_{j \beta}$. End of the protocol.

Check if search failed: If $S_{v^{*}}$ has the first Intra-MP-Address (null), there is no acquaintance of $S_{j \beta}$ in $M P_{v}$. End of the protocol.

Forward to next possible acquaintance: For any S-Node $S_{x \chi}$ having the Intra-MP-Address $\chi$ let $\Phi\left(S_{x \chi}\right)$ be the smallest natural number bigger null for that the equation $\chi$ modulo $d^{z-\Phi\left(\mathrm{S}_{\mathrm{x} \chi}\right)}=0$ holds.

$\operatorname{LetL}\left(S_{v^{*}}\right)$ be a subset of $\mathrm{B}\left(S_{v^{*}}\right)$ containing only those acquaintances $S_{v \chi}$ that have $\Phi\left(S_{v \chi}\right) \leq \Phi\left(S_{v^{*}}\right)$.
$S_{v^{*}}$ forwards $m$ to the S-Node of $\mathrm{L}\left(S_{v^{*}}\right)$ having the biggest Intra-MP-Address that is smaller than the Intra-MP-Address of $S_{v^{*}}$.That S-Node becomes the new $S_{v^{*}}$.

Continue with step 4).

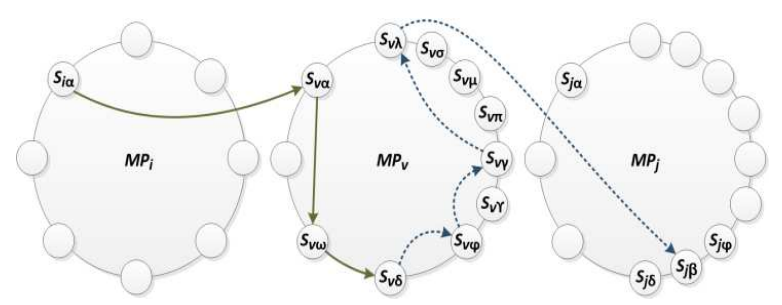

Figure 3. Searching a suboptimal preliminary acquaintance of Sj $\beta$ in MPv: The $S$-Nodes $S v Y, S v \pi$, Sv $\mu$ and $S v \sigma$ have been added later in the iterative process of making acquaintances [15] - after checking $S v \varphi$ and $S v \gamma$, it is clear that they cannot be acquaintances of Sjß so they may be skipped.

Let $F$ be the maximum number of forwarders required for partition-routing of a message split over $\mathrm{n}$ mistrust-parties. With the optimization, an upper bound for $F$ is:

$$
F \leq \sum_{i=0}^{n-1}\left(2 * F_{i}+3\right)
$$

Note: $F_{i}$ is the number of $\mathrm{S}$-Nodes required for partisan forwarding (4) within a single MP.

If the number of MPs is bigger than the security threshold $\Psi$, in case of any disturbance in some $M P_{k}$ it would be possible to avoid $M P_{k}$ completely and choose another MP instead to deliver a split message. Or if for the $Z n_{\Psi} n$ is chosen bigger than $\Psi$, in up to $n-\Psi$ different MPs there may be failures and the communication still works. Therefore, additional redundancy is superfluous for acquaintances in foreign MPs.

Let $G$ be the number of MPs. Any S-Node will not need more than $G-1$ acquaintances in all foreignMPs together.Per $\mathrm{S}$-Node belonging to $M P_{i}$, this leads to a total number $T A_{i}$ of required optimal acquaintances according to the following formula:

$$
T A_{i} \leq A_{i}+G-1=2 *\left\lceil\log _{d}\left(\# M P_{i}\right)\right\rceil+G-1(7)
$$

\section{Secure Communication for Users}

The solution presented so far is applicable only for secure communication between $\mathrm{S}$-Nodes which should always be online. Human beings and their client systems that are typically often offline should be able to communicate in a secure and reliable way with any $\mathrm{S}-\mathrm{Node}$, too.

Users having their own S-Node and who trust in its reliability could usetheir S-Node as a proxy server. Theproxy $\mathrm{S}$ - Node couldsimply forward messages addressing other S-Nodes- using the partition-routing protocol for those other $\mathrm{S}$-Nodes that are not acquaintances.

Of course, users could also manually exchange the required information for buildinga secure channel to additional S-Nodes belonging to other MPs with the S-Operators of these S-Nodes.Being able to build secure channels to at 
least $\Psi$ S-Nodes in $\Psi$ different MPs, the users could themselves start the partition-routing protocol. Hence, dependency on a single proxy S-Node being a potential point of failure could be avoided. The disadvantage would be the higher manual effort.

\section{Adaption for the S-Network}

The S-Network is a trustworthy repository currently developed at Fraunhofer FOKUS. The S-Network combines secure long term data storage and preservation in a computer network with non-repudiation and legal validity. For the future, the S-Network must guarantee to be secure even after any possible technical advance.

The S-Network uses MPs to store and maintain $2 * \Psi-$ 1 backup copies in a distributed way so that up to $\Psi-1 \mathrm{er}-$ roneous copies will not cause losses and that they can automatically be fixed. Secure communication between the systems storing the backup copies is essential. With the concept presented here, the MPs used for data preservation can be used again for the required message exchange and the same level of security and trustworthinesscan be guaranteed for both: Only if $\Psi$ or more MPs are incorrect there might be some unwanted incident that cannot be repaired.

Besides bit preservation, the S-Network also has to protect its content. For that access control, the same concepts including provable secure technologies like secret sharing and of course the same MPscan be used. Shares of a secret sharing split are distributed over systems in differentMPs so that at least threshold $\Psi$ of them have to cooperate to be able to reconstruct the plaintext.Combining the concepts for bit preservation and access control with MPs will require a significantly higher number of MPs than just $2 * \Psi-1$ because it must be possible to restore each share without making it easier to reconstruct the plaintext[16].

Secure communication with secret sharing between any two systems (i.e. S-Nodes) $S_{A}$ and $S_{B}$ in the S-Networkas shown before requires that either $S_{A}$ and $S_{B}$ are acquaintances or that partition-routing between them must be possible. Hence there must be at least $\Psi$ MPs in which both $S_{A}$ and $S_{B}$ have acquaintances. Since making acquaintances is a high manual effort, there should not be more acquaintances than absolutely necessary.

Agreeing on just $\Psi+1$ MPs in which any partisan forwarding should take place, each S-Node would need at most $\Psi+1$ foreign MP acquaintances -oneineach of these MPs. But the result would be unbalanced:S-Nodes belonging tothese "privileged"MPswould have amuch higher workload and more responsibility than the others.

\subsection{Multi-Partition-Routing}

Let $G$ be the total number of MPs. Choosing the foreign MPs in which an S-Node should haveacquaintances carefully and using an adapted routing protocol, secure communication between any two S-Nodes $S_{A}$ and $S_{B}$ is possible in a completely decentralized way with just $2 * \Psi$ foreign MP acquaintances per S-Nodefor any $G>2 * \Psi$.

Let theMPsbeindexed with natural numbers starting at zero. Each S-Node $S_{X}$ belonging to $M P_{y}$ should have acquaintances in those MPs having an index number $k=$ $(y+d)$ modulo $G$ for any $d \in \mathbb{Z} \backslash 0$ with $|d| \leq \Psi$.

$S_{X}$ needs alsoacquaintances in its own MPMP for optimized partisan forwarding in $M P_{y}$ as described above.If each S-Node has these acquaintances, the following multi-partition-routingprotocol offers secure communication between any S-Nodes $S_{A}$ belonging to $M P_{a}$ and $S_{B}$ belonging to $M P_{b}$ that are not acquaintances:

Preparation:As described in section 5.2.step 1).

Separation: $S_{A}$ sends each $\tau_{i}$ over a secure channel to a different foreign acquaintance $S_{f}$ not belonging to $M P_{b} . S_{A}$ may not send more than one $\tau_{i}$ into any $M P_{k}$.

Check and forwarding: Each forwarding S-Node $S_{f}$ decrypts and checks messages $m$ arriving over secure channels from its foreign acquaintances. If $m$ does not contain an identity confirmation $I_{A i}$ (i.e. $m=\tau_{i}$ ) then $S_{f}$ adds $I_{A i}$ as proof of authenticity to $m: m=\tau_{i} \circ I_{A i}$.

Let $k$ be the index of the MP $S_{f}$ belongs to.Let $p$ be the index of the MP from which $m$ was send to $S_{f}$.

If $S_{f}$ has an acquaintancein $M P_{b}$ thencontinue for $m$ with the partition-routing protocol(section 5.2)startingwithstep 3 )b) if $S_{B}$ is an acquaintance of $S_{f}$ or with step 3)a) otherwise. End of this protocol.

Calculate the directiond: If $((0<k-p \leq \Psi) \mathrm{V}$ $(0<G+k-p \leq \Psi))$ thend $=1$, else $d=-1$.

Calculate shortforwards only width $w$ and border index $s$ : Let $w$ be the smallest natural number with $(a-d *$ $w)_{\text {modulo }} G=b$. Let $h$ be the biggest natural number with $(h * \Psi)_{<} G-\Psi$.Then the border index $s$ is: $s=(a+d *$ $h * \Psi)$ modulo $G$.

Calculate index $q$ of the next forwarder's MP: If anintegert with $|t|<\Psi$ and $s=(k+\mathrm{t}){ }_{\text {modulo }} G$ exists then $\Delta=$ welse $\Delta=\Psi$.

$q=(k+d * \Delta)_{\text {modulo }} G$.

$\mathrm{S}_{\mathrm{f}}$ chooses an acquaintance belonging to $M P_{q}$ as next forwarder and sends $m$ over a secure channel to that S-Node. Do step III.for the next forwarder.

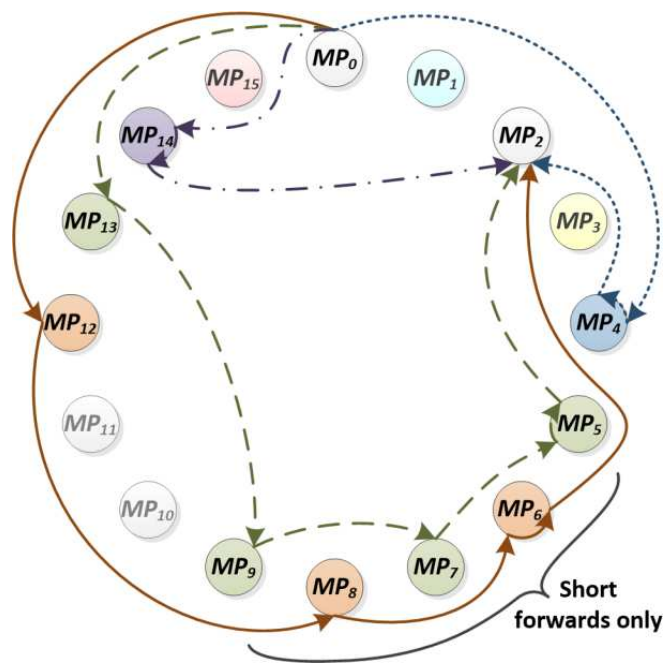

Figure 4. Multi-partition-routing with $G=16$ and $\Psi=4$.

The multi-partition-routingprotocol makes sure that no 
single MP except $M P_{b}$ receives more than one piece of $\mathrm{Z} n_{\Psi}\left(x_{p}\right)$.The sender $S_{A}$ initially separates the $\tau_{i}$ in step II.

As soon as $\tau_{i}$ arrives in an MP having an acquaintance in $M P_{b}$ (step III.i), only partisan forwarding and a direct forwarding to $S_{B}$ are performed for $\tau_{i}$ till the protocol ends.

In step III.v $\tau_{i}$ is forwarded to some S-Node belonging to a foreign MP that is not $M P_{b}$-and since the protocol continues with step III, this might happenmultiple times. Each $\tau_{i}$ goes either only increasingmodulo $G$ through the MP indices $(d=1)$ or onlyin the other $\operatorname{direction}(d=-1)$.In each direction there are at most $\Psi$ different shares.Since all the shares routed in the same directionarein different initial MPs after step II andsince the distance modulo $G$ between any two indices of these initial MPs is lower than $\Psi$, shares forwarded in the same direction stay separated as long as the index is decremented or incremented by $\Delta=\Psi$.

It can be shown that $\Delta>\Psi$ is never applied. $\Delta<$ $\Psi$ might beapplied if $S_{A}$ has acquaintances in $M P_{b}$ to ensure that no share is forwarded in step III.v to an MP in which $S_{A}$ also has acquaintances. The border $s$ is the last index for which applying $\Delta=\Psi$ is safe. When passing sandif there are no acquaintances in $M P_{b}$, only short forwards with $\Delta=w$ are applied. This can happen only for up tow shares since at most the S-Nodes in $w$ initial MPs have no acquaintances in $M P_{b}$. As those winitial MPs have indices that are consecutive modulo Git is straightforward to show thatseparation is preserved.

Multi-partition-routing requiresfewer acquaintancesin foreign MPs.But there may be up to $G / \Psi+\Psi$ additional forwarders required for each $\tau_{i}$. An upper bound $F_{m}$ for the total number of forwarders in multi-partition-routing using $n$ shares can be defined in a similar manner asin (6).

$$
F_{m} \leq \sum_{i=0}^{n-1}\left(2 * F_{i}+3+\left(\frac{G}{\Psi}+\Psi\right)\right)
$$

\section{Conclusion}

This article presents a concept for secure communication in large computer networks without a "trusted party" or a "web of trust" and without relying on assumptions of complexity theory. Unlike in previous PSMT proposals, a realistic concept to actually create communication paths with disjunct sets of nodes is provided.Depending on the choice of algorithms used to build secure channels between acquaintances, even unlimited calculating power cannothelp to break the security of the concept.Hence the solution is applicable wherestrong long term security is required.

Perfect security is not guaranteed: Attackers could randomly generate a message passing integrity tests. The likelihood that this happens can be reduced by expanding the MAC.The security also depends on the choice of $\Psi$ : A manipulation involving at least $\Psi$ S-Nodes in $\Psi$ different MPs can break the concept. Increasing $\Psi$ and the number of MPs probably only makes sense up to a certain degree.It is crucial to prevent manipulative cooperation among the MPs.

For a trustworthy repository that has to guarantee secure long term serviceability like the S-Network, the solution presented here seems to be a good choice. Using the same concepts and the same MPsto provide bit preservation, secrecy and access control, the S-Network will need more different MPs than the number of MPs actually required for secure communication. Multi-partition-routing introduced in this article keeps the required number of acquaintances in foreign MPs on a low level even in such scenarios, producing only moderately increased forwarding effort and slightly longer message transmission routes.

\section{References}

[1] AmitabhaBagchie et al.: Constructing Disjoint Paths for Secure Communication; Lecture Notes in Computer Science, 2003, Volume 2848/2003 pp. 181-195; Springer 2003

[2] Paul Baran: On Distributed Communications Networks; RAND Corporation Santa Monica 1962; http://www.rand.org/pubs/papers/P2626 (2010-01-25)

[3] Alex Biryukov, Dmitry Khovratovich: Related-key Cryptanalysis of the Full AES-192 and AES-256; Cryptology ePrint Archive 2009; http://eprint.iacr.org/2009/317

[4] J. Callas et al.: OpenPGP Message Format; The Internet Society 2007; http://tools.ietf.org/html/rfc4880 (2011-02-02)

[5] Joan Daemen, Vincent Rijmen: The design of Rijndael: AES - theadvanced encryption standard; Springer 2002

[6] D. Dolev, C. Dwork, O. Waarts, M. Yung: Perfectly secure message transmission; 31st Annual Symposium on Foundations of Computer Science (FOCS 1990) 1990

[7] Niels Ferguson, Bruce Schneier, Tadayoshi Kohno: Cryptography Enineering; Wiley Publishing, Indianapolis 2010

[8] IBM Research Division; IBM's Test-Tube Quantum Computer Makes History; First Demonstration Of Shor's Historic Factoring Algorithm; ScienceDaily 2001

[9] J. Kohl, C. Neuman: The Kerberos Network Authentication Service; Massachusetts Institute ofTechnology 1993; www.ietf.org/rfc/rfc1510.txt(2011-02-02)

[10] RSA Laboratories: PKCS \#1 v2.1: RSA Cryptography Standard; RSA Security Inc. 2002; $\mathrm{ftp}: / / \mathrm{ftp}$. rsasecurity.com/pub/pkcs/pkcs-1/pkcs-1v2-1.p df (2011-02-02)

[11] Adi Shamir: How to share a secret; Communications of the ACM v.22 issue 11 pp. 612-613; ACM New York 1979

[12] C. E. Shannon: Communication theory of secrecy systems; Bell System Technical Journal 28 pp. 656 - 715; Bell Labs 1949

[13] Peter W. Shor: Polynomial time algorithms for prime factorization and discrete logarithms on a quantum computer; Bell Labs 1994

[14] Alexander Sotirov et al.: MD5 considered harmful today: Creating a rogue CA certificate; 25 th Annual Chaos Communication Congress in Berlin 2008

[15] J.Viehmann: Secure communication with secret sharing in static computer networks with partition in mistrust-parties, 
proc. of the NinthAnnual Conference on Privacy, Security and Trust (PST)Montreal,pp. 205-212, IEEE 2011

[16] J.Viehmann: The Theory of Creating Trust with a Set of Mistrust-Parties and its Exemplary Application for the
S-Network, proc. of the Tenth Annual Conference on Privacy, Security and Trust (PST) Paris, pp. 185-194, IEEE 2012 